\title{
Persistent spatial maze-learning deficits in hippocampal-lesioned rats across a 7-week postoperative period
}

\author{
DANIEL P. KIMBLE and EUGENE DANNEN \\ University of Oregon, Eugene, Oregon 97403
}

\begin{abstract}
Rats with bilateral dorsal hippocampal lesions were tested on a series of three RabinovitchRosvold maze patterns at either 1 week (Days 6, 7, and 8) or 7 weeks (Days 48, 49, and 50) following surgery. All rats in both groups were significantly impaired in the acquisition of these complex spatial maze patterns, regardless of the postoperative delay prior to testing. This lack of improvement stands in sharp contrast to earlier findings from our laboratory, in which it was determined that the decrease in levels of spontaneous alternation and the alterations in open-field activity produced by hippocampal lesions did return to normal levels within 6 weeks following surgery. The present results indicate that spatial maze learning may be classified as a persistent (although perhaps not permanent) deficit produced by hippocampal damage.
\end{abstract}

Recent evidence indicates that in some brain regions, neurons deprived of their normal input through injury undergo considerable reinnervation from remaining intact neurons (Lynch \& Cotman, 1975; Raisman, 1969; Raisman \& Field, 1973; Steward, Cotman, \& Lynch, 1974, 1976). In particular, vacated synaptic sites which appear in the septal area following fimbrial lesions appear to be filled in or "reclaimed" during the first few postoperative weeks, presumably from remaining, intact axons which ascend in the medial forebrain bundle (Moore, Bjorkland, \& Stenevi, 1971, 1974; Raisman, 1969; Raisman \& Field, 1973).

Relatively little information is available regarding the possible functional significance of such reinnervation. Electrophysiological evidence indicates that reinnervation of the dentate gyrus by contralateral projections from the entorhinal cortex is accompanied by a new short-latency evoked potential in the dentate gyrus to contralateral entorhinal cortex stimulation. This activity is due to monosynaptic activation of the previously denervated dentate granule cells by the reinnervating contralateral entorhinal fibers (Steward et al., 1974).

Recently, we found that following dorsal hippocampal lesions, two different behaviors, spontaneous alternation and open-field activity, were altered by the lesion, but that both behaviors returned to near-normal levels after the first 2 postoperative weeks (Kimble, 1976). Testing of these rats continued across a 6-week postoperative period, with continued normal levels of these behaviors. This postoperative time course coincided well with that reported for septal reinnervation (Moore et al., 1974; Raisman, 1969). While no direct relationship between these behavioral changes and the reported anatomical changes could be made on the basis of that report, the results were compatible with the concept that such reinnervation in the adult rat brain may be accompanied by the return of lesion-induced behavioral changes toward normal levels. The present results indicate that there may be limitations to this behavioral recovery.

In our earlier report (Kimble, 1976), both of the behaviors which were examined were "natural," or untrained, behaviors. Those behaviors were chosen for examination because they had been repeatedly found to be altered with hippocampal lesions, and because they seem to be quite insensitive to "learning" effects with repeated measurements. In the present experiment, we turned to a learned behavior-complex spatial maze acquisition, which has also repeatedly been found to be impaired by hippocampal damage (Gross, Chorover, \& Cohen, 1965; Hostetter \& Thomas, 1967; Hughes, 1965; Kaada, Rasmussen, \& Kviem, 1961; Kimble, 1963; Madsen \& Kimble, 1965; Thomas, 1971). We examined the performance of rats on complex maze problems at 1-week and at 7-week postoperative periods. Unlike our findings with spontaneous alternation and openfield activity, we found a persistent deficit in the acquisition of these maze problems following bilateral hippocampal lesions, with no indication of any behavioral recovery across the time period investigated. The implications of these and other findings are discussed.

\section{METHOD}

Animals
The subjects were 32 male Sprague-Dawley CD (Random-
bred) albino rats purchased from the Charles River Co.,
Wilmington, Massachusetts. They were approximately 100 to
120 days of age at the beginning of the experiment. Sixteen 
of the rats received bilateral lesions to the dorsolateral hippocampus. The remaining 16 rats served an unoperated controls. All operated animals survived the operation and all 32 rats completed all phases of the experiment.

\section{Surgery and Histology}

All operations were performed by one of us (D.P.K.) under clean conditions. Lesions were performed in one-stage by aspiration under visual control, using a procedure described previously (Kimble, 1963). Animals were anesthetized with Nembutal $(52 \mathrm{mg} / \mathrm{kg})$. Each animal received an IM injection of a broadspectrum antibiotic shortly after surgery. Following completion of the experiment, the animals were sacrificed with an overdose of Nembutal. The brains were removed, fresh frozen, sectioned at 12 microns, and stained with thionin according to the procedure developed by BreMiller (1971).

\section{Apparatus}

The apparatus used was a Hebb-Williams closed field, with maze patterns developed by Rabinovitch and Rosvold (1951). The main field of the maze measured $78.7 \times 78.7 \mathrm{~cm}$ and had 11.4-cm-high walls. Attached to the main maze field were a goalbox and startbox, both measuring $30 \mathrm{~cm}$ long, $15 \mathrm{~cm}$ high, and $13.5 \mathrm{~cm}$ wide. A sheet of clear Plexiglas served as a cover for the main maze field, and the goal- and startboxes were equipped with wire screen covers and wooden guillotine-type doors. Wooden inserts constructed of plywood were used to construct the various maze patterns. The main maze field, goal- and startboxes, and wooden inserts were all painted a flat, medium gray. The guillotine doors were unpainted brown fiberboard.

\section{Procedure}

The animals were housed individually and maintained on an ad-lib food schedule. Twelve days prior to operation, all rats were placed on a 24-h water-deprivation schedule. Pretraining began on the following day. For 3 days, rats were placed in the maze, in groups of four, with no inserts present for $15 \mathrm{~min}$ each day. Following this group maze acclimation procedure, each animal was given 3 consecutive days of individual maze training, using Practice Problems A, B, and C (Rabinovitch \& Rosvold, 1951). About 10-15 sec of drinking was allowed after each successful run. At the end of the pretraining procedures, all rats were running rapidly to the goalbox for water.

The room in which they were housed and trained was on a 12-12 h light-dark schedule. The light cycle began at 6:00 a.m. Maze training was done in dim illumination early in the dark cycle, between 7:00 and 9:00 p.m. No significant illness occurred in any of the rats during the duration of the experiment.

Maze-training: One-week group. Eight hippocampal-lesioned rats and eight controls formed the 1-week group. Each lesioned rat, along with its matched control, was tested on Maze Problems 2, 6, and 11 of the Rabinovitch-Rosvold series on Days 6, 7 , and 8 following surgery. The floor plan of each of these problems is shown in Figures 1-3. Each rat received seven trials on each of the mazes. Trials were run consecutively, with 15 -sec access to a water bottle available in the goalbox following entry. If a rat did not make an entry into the goalbox within $3 \mathrm{~min}$, that trial was terminated and the next trial begun.

Seven-week group. The other 16 rats (8 hippocampallesioned and 8 controls) were treated in the same fashion as the 1-week group, except that 7 weeks were allowed to elapse between the operation and the maze training. Thus, these rats were trained on Maze Pattern 2 on Day 48 postoperatively, on Maze Pattern 6 on Day 49, and on Maze Pattern 11 on Day 50. In all other respects they were treated in an identical fashion to the 1-week rats.

The animals in the 1-week group were also tested on a second series of maze problems on Days 48,49 , and 50 postoperatively. This series was composed of Maze Problems 1, 5, and 10. Testing procedures were otherwise the same.

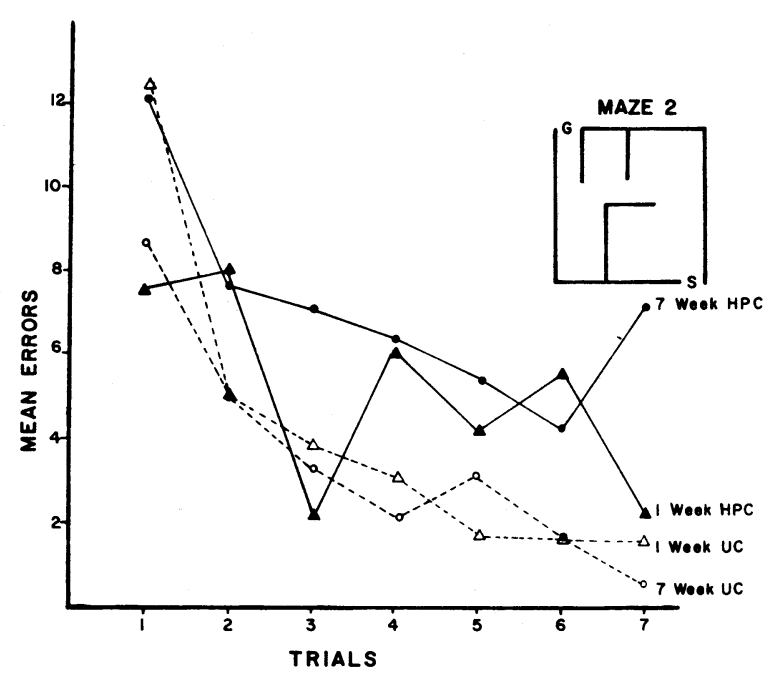

Figure 1. Mean errors on the seven trials on Maze Pattern 2 from the Rabinovitch-Rosvold series. A 1-week groups (triangles) were tested on Day 6 postoperatively. The 7-week groups (circles) were tested on Day 48 postoperatively. The maze pattern is illustrated in the upper right-hand corner. Abbreviations: HPC = hippocampal-lesioned, $\mathrm{UC}=$ unoperated controls, $\mathrm{S}=$ startbox, $\mathbf{G}=$ goalbox.

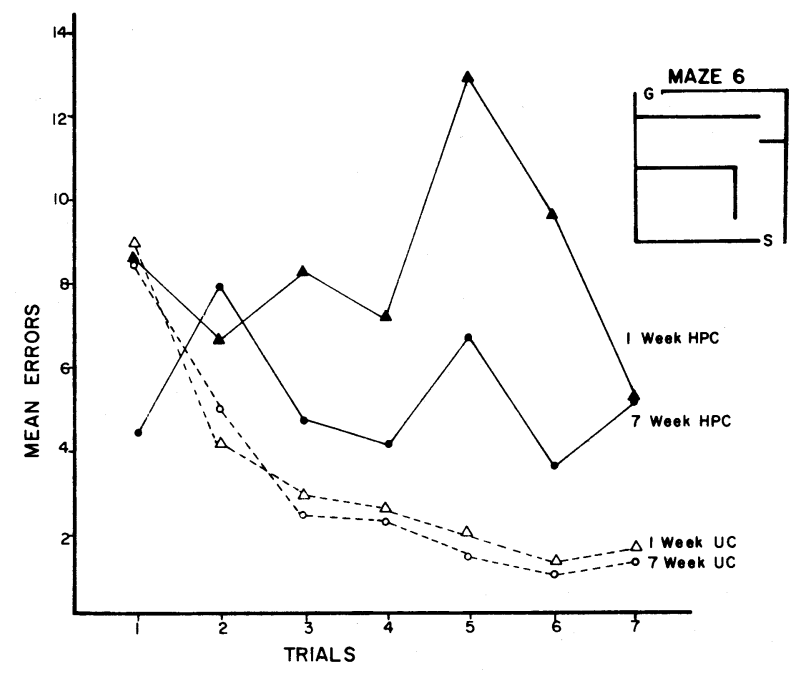

Figure 2. Mean errors on the seven trials of Maze Pattern 6 from the Rabinovitch-Rosvold series. The 1-week groups (triangles) were tested on Day 7 postoperatively. The 7 -week groups (circles) were tested on Day 49 postoperatively. The maze pattern is illustrated in the upper right-hand corner. Abbreviations are the same as in Figure 1.

\section{RESULTS}

\section{Behavior}

The hippocampal-lesioned rats from both the 1-week and 7-week experiments showed a significant impairment in the acquisition of the maze patterns. Table 1 gives group data from these experiments. Differences between the groups were evaluated using the Mann- 


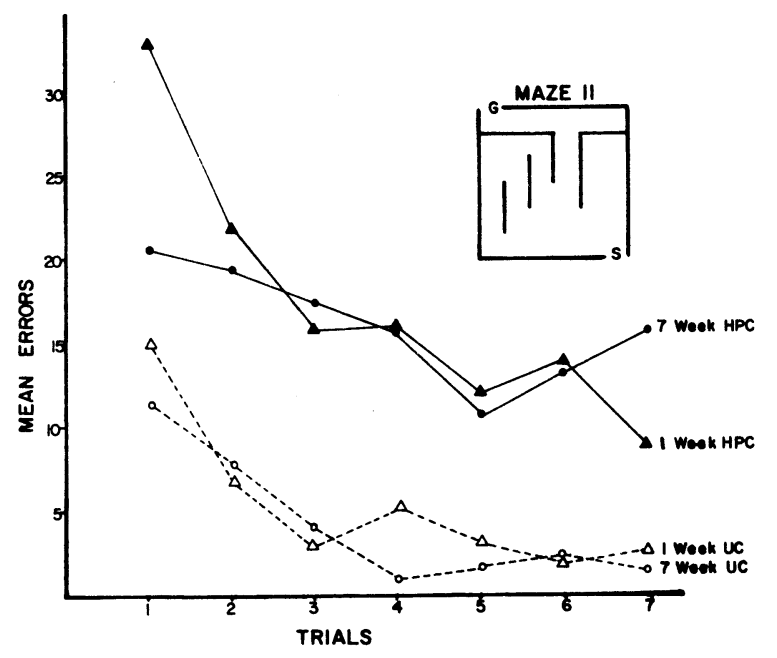

Figure 3. Mean errors on the seven trials of Maze Pattern 11 from the Rabinovitch-Rosvold series. The 1-week groups (triangles) were tested on Day 8 postoperatively. The 7 -week groups (circles) were tested on Day 50 postoperatively. The maze pattern is illustrated in the upper right-hand corner. Abbreviations are the same as in Figures 1 and 2.

Whitney U test (Siegel, 1956). With the single exception of the initial maze pattern (Pattern 2) in the 1-week group, the hippocampal-lesioned rats committed significantly more errors on all the maze patterns than did the controls. See Figures 1-3. The lesioned subjects made about 2.5 times as many total errors as did their controls across the series of three maze patterns. This was true for both the 1-week and the 7-week groups. No statistically significant differences were obtained between the 1-week and 7-week performances, for either the hippocampal-lesioned or the control animals.

The 1-week subjects were also tested on a second series of maze patterns (Patterns 1, 5, 10) from the Rabinovitch-Rosvold series. The hippocampal-lesioned rats made significantly more errors on each of these three maze patterns than did the controls (see Table 2). These 1-week animals were also retested on Maze 11 . Control rats made fewer errors on the retention test than they had done on the initial testing with this maze pattern, as did seven of the eight hippocampal-lesioned rats. Nevertheless, the hippocampal-lesioned rats still made significantly more errors on this retention test than did the control animals.

Maze performance of all groups on individual maze patterns was compared with total series errors using a Spearman rank correlation coefficient (Siegel, 1956). Eighteen such correlations were computed, of which seven reached statistical significance at the .05 level or better. For the 1-week control animals, the highest correlation was between their ranks on errors on Maze 11 and total errors across the series of three patterns $\left(\mathrm{r}_{\mathrm{s}}=\right.$ $.881, \mathrm{p}=.01)$. On the second series of maze patterns on which these animals were tested, the only statistically significant correlation was found for ranks on errors on Maze Pattern 5 and total series errors $\left(r_{s}=.940\right.$, $\mathrm{p}=.01$ ). For the control animals in the 7-week group, two statistically significant correlations were found for rank orders: Maze Pattern 2 errors and total series errors $\left(r_{s}=.740, p=.05\right)$ and Maze Pattern 11 errors and total

Table 1

Mean Number and Range of Errors on Three Rabinovitch-Rosvold Mazes by Hippocampal-Lesioned (HPC) and Unoperated (UC) Rats 1 or 7 Weeks Postoperatively

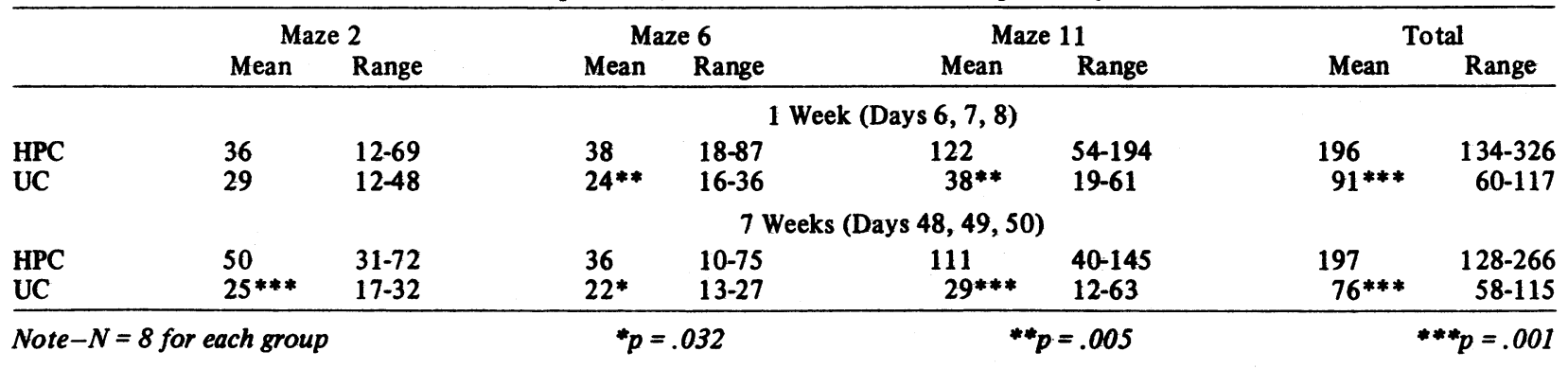

Table 2

Mean Number and Range of Errors on Second Battery of Three Rabinovitch-Rosvold Mazes by Hippocampal-Lesioned (HPC) and Unoperated (UC) Rats, 7 Weeks Postoperatively

\begin{tabular}{|c|c|c|c|c|c|c|c|c|c|c|}
\hline & \multicolumn{10}{|c|}{ Maze Problems } \\
\hline & \multicolumn{2}{|c|}{1} & \multicolumn{2}{|c|}{5} & \multicolumn{2}{|c|}{10} & \multicolumn{2}{|c|}{ Total } & \multicolumn{2}{|c|}{11 (Retention) } \\
\hline & Mean & Range & Mean & Range & Mean & Range & Mean & Range & Mean & Range \\
\hline $\begin{array}{l}\text { HPC } \\
\text { UC }\end{array}$ & $\begin{array}{l}133 \\
10^{* *}\end{array}$ & $\begin{array}{c}83-208 \\
6-27\end{array}$ & $\begin{array}{l}45 \\
21^{*}\end{array}$ & $\begin{array}{r}29-78 \\
9-46\end{array}$ & $\begin{array}{l}69 \\
20^{* *}\end{array}$ & $\begin{array}{l}24-148 \\
14-39\end{array}$ & $\begin{array}{l}247 \\
51^{* *}\end{array}$ & $\begin{array}{c}165-387 \\
35-94\end{array}$ & $\begin{array}{l}66 \\
20^{* *}\end{array}$ & $\begin{array}{c}32-122 \\
9-28\end{array}$ \\
\hline
\end{tabular}


series errors $\left(r_{s}=.786, p=.05\right)$. For the hippocampallesioned rats in the 7-week group, a significant correlation was found only for errors on Maze Pattern 11 and total series errors $\left(\mathrm{r}_{\mathrm{s}}=.714, \mathrm{p}=.05\right)$.

Among the 1-week hippocampal-lesioned rats, there were no statistically significant correlations on the first series of maze patterns. On the second series, statistically significant rank order correlations were obtained for ranks on errors on Maze Pattern 6 and total series errors $\left(r_{s}=.643, p=.05\right)$ and Maze Pattern 11 and total series errors $\left(\mathrm{r}_{\mathrm{s}}=.714, \mathrm{p}=.05\right)$.

\section{Histology}

The lesions were quite uniform in extent and location, conforming well to histological results published earlier (Kimble, 1976). The surgery resulted in substantial ablation of the dorsal and dorsolateral hippocampus and dentate gyrus, with occasional sparing of some medial and anterior portions of these structures, but invariably severing the hippocampal formation at some point on both sides. The fimbria was uniformly involved in the lesions, but some residual fimbrial tissue remained in most brains, typically bilaterally. Stria terminalis was undamaged in all brains. Some entorhinal cortex damage was incurred in some animals, and the subiculum was typically partially involved. The ventral and posterior portions of the hippocampal formation were spared in all animals, in amounts which were estimated to vary from approximately $25 \%$ to $35 \%$ of the total hippocampal volume.

\section{DISCUSSION}

In an earlier experiment (Kimble, 1976), it was found that spontaneous alternation levels and activity in the open field returned to normal levels after initial lesioninduced changes within the first 2-3-week postoperative period. In the present experiment, we found no evidence of recovery of the lesion-induced maze acquisition deficit. It is possible that further recovery time would have given a different result, but the anatomical findings suggest that the reinnervation process in the septal area begins a few days following surgery and is quite marked by 30 days postoperatively. According to Raisman (1969), by 42 days postoperatively: "the general configuration of synapses has reached a stable state." While Raisman's data regarding reinnervation in the septal area following hippocampal-fimbrial damage are clearly more relevant to the present report, Illis (1973) has reported anatomical evidence that synaptic changes in the spinal cord of the cat may continue for as long as 16 to 20 weeks following dorsal root section. Thus, while we cannot state with certainty that no recovery of the maze acquisition deficit can occur in rats with hippocampal lesions, we find no evidence for such recovery either. Olton (1977) has recently published similar findings, using an eight-arm spatial maze. Unlike the reduction in levels of spontaneous alternation and marked changes in open-field locomotion which are produced by this same lesion, the maze acquisition deficit can now be identified as a "persistent" (although perhaps not "permanent") behavioral effect of hippocampal damage. These findings are consistent with results of unit recordings from the hippocampus (O'Keefe \& Dostrovsky, 1971) and with the hypothesis that the hippocampus is necessary for spatial orientation and/or spatial memory in the rat (Olton, 1977).

Finally, it is interesting to note that some recovery of a very similar behavioral deficit using RabinovitchRosvold maze patterns has been reported following surgical interruptions in the perforant paths which connect the dentate gyrus with the entorhinal cortex. This behavioral recovery of maze learning ability was noted on Days 34-36 postoperatively (Myhrer, 1975). Thus, the hippocampal formation itself would seem to be critically involved in spatial maze acquisition, while damage to the neural connections with the entorhinal cortex may be compensated for through reinnervation (or other) processes during the first weeks following injury.

Whether other behavioral effects of hippocampal lesions will prove to be transitory, persistent, or permanent is a subject for future research. Such research should further enlarge our knowledge regarding the functional significance of reinnervation processes.

\section{REFERENCES}

BreMiller, R. A. A rapid technique of preparing frozen sections of small brains. Physiology \& Behavior, 1971, 6, 463-464.

Gross, C. G., Chorover, S. L., \& Cohen, S. M. Caudate, cortical, hippocampal and dorsal thalamic lesions in rats: Alternation and Hebb-Williams maze performance. Neuropsychologia, 1965, 3, 53-68.

Hostetter, G., \& Thomas, G. J. Evaluation of enhanced thigmotaxis as a condition of impaired maze learning by rats with hippocampal lesions. Journal of Comparative and Physiological Psychology, 1967, 63, 105-110.

Hughes, K. R. Dorsal and ventral hippocampus lesions and maze learning: Influence of preoperative environment. Canadian Journal of Psychology, 1965, 19, 325-331.

ILLIS, L. S. Experimental model of regeneration in the central nervous system I: Synaptic changes. Brain, 1973, 96, 47-60.

KaAda, B. R., Rasmussen, E. W., \& Kviem, O. Effects of hippocampal lesions on maze learning and retention in rats. Experimental Neurology, 1961, 3, 333-355.

KImbLe, D. P. The effects of bilateral hippocampal lesions in rats. Journal of Comparative and Physiological Psychology, 1963, 56, 273-283.

Kimble, D. P. Changes in behavior of hippocampal-lesioned rats across a 6-week postoperative period. Physiological Psychology, 1976, 4, 289-293.

LyNCH, G., \& Cotman, C. W. The hippocampus as a model for studying anatomical plasticity in the adult brain. In $R$. L. Isaacson \& K. H. Pribram (Eds.), The hippocampus. New York: Plenum, 1975. Pp. 123-154.

Madsen, M. C., \& Kimble, D. P. The maze behavior of hippocampectomized rats under massed and distributed trials. Psychonomic Science, 1965, 3, 193-194.

Moore, R. Y., Bjorkland, A., \& Stenevi, U. Plastic changes in 
the adrenergic innervation of the rat septal area in response to denervation. Brain Research, 1971, 33, 13-35.

Moore, R. Y., Bjorkland, A., \& Stenevi, U. Growth and plasticity of adrenergic neurons. In F. O. Schmitt \& F. G. Worden (Eds.), The neurosciences, third study program. Cambridge, Mass: MIT Press, 1974. Pp. 961-977.

MYHRER, T. Maze performance in rats with hippocampal perfor ant path lesions: Some aspects of functional recovery. Physiology and Behavior, 1975, 15, 433-437.

O'KeEfE, J., \& Dostrovsky, J. The hippocampus as a spatial map. Preliminary evidence from unit activity in the freely-moving rat. Brain Research, 1971, 34, 171-175.

Otron, D. S. Spatial memory. Scientific American, 1977, 236, 82-98.

Rabinovitch, M. S., \& Rosvold, H. E. A closed-field intelligence test for rats. Canadian Journal of Psychology, 1951, 5, 122-128.

RaIsman, G. Neuronal plasticity in the septal nuclei of the adult rat. Brain Research, 1969, 14, 25-48.

Raisman, G., \& Field, P. M. A quantitative investigation of the development of collateral reinnervation after partial deafferentation of the septal nuclei. Brain Research, 1973, 50, 241-264.
SIEGEL, S. Nonparametric statistics for the behavioral sciences. New York: McGraw-Hill, 1956.

Steward, O., Cotman, C. W., \& Lynch, G. S. Growth of a new fiber projection in the brain of adult rats: Re-innervation of the dentate gyrus by the contralateral entorhinal cortex following ipsilateral entorhinal lesions. Experimental Brain Research, 1974, 20, 45-66.

Steward, O., Corman, C., \& Lynch, G. A quantitative autoradiographic and electrophysiological study of the reinnervation of the dentate gyrus by the contralateral entorhinal cortex following ipsilateral entorhinal lesions. Brain Research, 1976, 114, 181-200.

Thomas, G. J. Maze retention by rats with hippocampal lesions and with fornicotomies. Journal of Comparative and Physiological Psychology, 1971, 75, 41-49.

(Received for publication June 2, 1977; revision accepted September 12, 1977.) 